\title{
Flavor structure in D-brane models: Majorana neutrino masses
}

\author{
Yuta Hamada, Tatsuo Kobayashi and Shohei Uemura \\ Department of Physics, Kyoto University, \\ Kyoto 606-8502, Japan \\ E-mail: hamada@gauge.scphys.kyoto-u.ac.jp, \\ kobayashi@gauge.scphys.kyoto-u.ac.jp, \\ uemura@gauge.scphys.kyoto-u.ac.jp
}

ABSTRACT: We study the flavor structure in intersecting D-brane models. We study anomalies of the discrete flavor symmetries. We analyze the Majorana neutrino masses, which can be generated by D-brane instanton effects. It is found that a certain pattern of mass matrix is obtained and the cyclic permutation symmetry remains unbroken. As a result, trimaximal mixing matrix can be realized if Dirac neutrino mass and charged lepton mass matrices are diagonal.

KEYWORDS: Intersecting branes models, Discrete and Finite Symmetries, Anomalies in Field and String Theories, Nonperturbative Effects

ARXIV EPRINT: 1402.2052 


\section{Contents}

1 Introduction 1

2 Discrete flavor symmetries $\quad 2$

3 Discrete anomalies $\quad 4$

$3.1 \mathrm{U}(1)$ anomalies 4

$\begin{array}{lll}3.2 & \text { Discrete anomalies } & 6\end{array}$

4 Majorana neutrino masses $\quad 7$

$\begin{array}{lll}4.1 & \text { Neutrino mass matrix } & 7\end{array}$

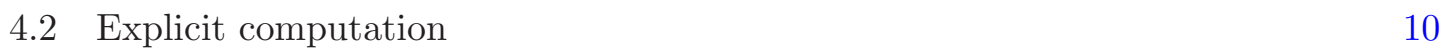

$\begin{array}{lll}4.3 & \text { Phenomenological implication } & 12\end{array}$

5 Conclusion and discussion $\quad 13$

A Integration of Wilson line moduli $\quad 13$

\section{Introduction}

The Standard Model has been confirmed by the discovery of the Higgs scalar and other precision measurements. However, it has various mysteries still. One of them is the mystery on the flavor structure. Why are there three generations? Why are quark and lepton masses hierarchical? Which mechanism determines their mixing angles? Indeed, the Yukawa sector has most of free parameters in the Standard model. Discrete flavor symmetries would be important to understand fermion masses and mixing angles [1-5]. For example, the mixing matrix in the lepton sector, the PMNS matrix, can be approximated by the tribimaximal mixing matrix in the limit $\theta_{13}=0$ [6-8]. In field-theoretical model building, one starts with a large flavor symmetry. Then, one assumes that the flavor symmetry breaks properly into $Z_{3}$ and $Z_{2}$ subsymmetries in the charged lepton or the neutrino masses, such that the tri-bimaximal mixing can be realized.

Superstring theory is a promising candidate for unified theory of all of the interactions including gravity and all of the matter fields and Higgs field(s) (see for a review [9]). It is found that superstring theory on six-dimensional compact space leads to interesting flavor structures. In particular, certain types of four-dimensional superstring models with rather simple six-dimensional compact spaces such as tori and orbifolds lead to definite discrete flavor symmetries. For example, intersecting D-brane models and magnetized Dbrane models are among interesting model building in superstring theory [10-16] (see for review $[9,17]$ and references therein). These intersecting/magnetized D-brane models can 
lead to discrete flavor symmetries such as $D_{4}, \Delta(27), \Delta(54)[18-22] .{ }^{1}$ Similar discrete flavor symmetries can be derived in heterotic string theory on orbifolds [23-25]. ${ }^{2}$ In these models, we can calculate explicitly Yukawa couplings and higher order couplings [27-39].

However, such discrete flavor symmetries may be broken by non-perturbative effects. From such a viewpoint, anomalies of discrete symmetries [44, 46-49] are important because anomalous symmetries may be broken by non-perturbative effects. Even anomaly-free U(1) gauge symmetries can be broken when axions couple with $\mathrm{U}(1)$ gauge bosons and they become massive. Furthermore, as concrete non-perturbative effects, D-brane instanton effects have been studied [50] (see also for a review [51] and references therein). From the viewpoint of flavor physics, one of important points is that D-brane instanton effects can generate right-handed Majorana neutrino masses [52-55]. Then, it is also important to investigate patterns of right-handed Majorana neutrino mass matrices derived by D-brane instanton effects and study whether such effects break some or all of discrete flavor symmetries and which symmetries remain unbroken.

In this paper, we study the flavor structure in intersecting D-brane models as well as magnetized D-brane models. We study anomalies of discrete flavor symmetries derived in intersecting D-brane models. We also study right-handed Majorana neutrino mass matrices, which can be generated by D-brane instanton effects. We show which types of Majorana mass matrices can be derived and which flavor symmetries remain unbroken even with right-handed Majorana neutrino mass matrices generated by D-brane instanton effects.

This paper is organized as follows. In section 2, we review briefly the discrete flavor symmetries derived from intersecting D-brane models as well as magnetized D-brane models. In section 3, we study anomalies of these discrete flavor symmetries. In section 4, we study right-handed Majorana masses generated by D-brane instanton effects. Section 5 is devoted to conclusion and discussion. In appendix A, we show the computation to integrate non-vanishing Wilson line phase.

\section{Discrete flavor symmetries}

In this section, we review briefly discrete flavor symmetries appearing in intersecting Dbrane models as well as magnetized D-brane models [18, 21, 22]. For concreteness, we consider IIA D6-brane models on $T^{6}=T_{1}^{2} \times T_{2}^{2} \times T_{3}^{2}$, where each D6-brane wraps one-cycle of each $T^{2}$ of $T^{6}=T_{1}^{2} \times T_{2}^{2} \times T_{3}^{2}$. That is, our setup is as follows. We consider $N_{a}$ stacks of D6-branes, which lead to $\mathrm{U}\left(N_{a}\right)$ gauge symmetry, and they have winding numbers $\left(n_{a}^{i}, m_{a}^{i}\right)$ along the $x_{i}$ and $y_{i}$ directions on $T_{i}^{2}$, where we use orthogonal coordinates $\left(x_{i}, y_{i}\right)$ on $T_{i}^{2}$. When we denote the basis of one-cycles on $T_{i}^{2}$ by $\left[a_{i}\right]$ and $\left[b_{i}\right]$, which correspond to the $x_{i}$ and $y_{i}$ directions, the three-cycle, along which this set of D6-brane winds, is represented by

$$
\left[\Pi_{a}\right]=\prod_{i=1}^{3}\left(n_{a}^{i}\left[a_{i}\right]+m_{a}^{i}\left[b_{i}\right]\right) .
$$

\footnotetext{
${ }^{1}$ See also [26].

${ }^{2}$ See for recent works on other discrete stringy symmetries, e.g. [40-47] .
} 
Here, we consider two sets of D-branes, one set is $N_{a}$ stacks of D6-branes and another is $N_{b}$ stacks of D6-branes. These lead to $\mathrm{U}\left(N_{a}\right) \times \mathrm{U}\left(N_{b}\right)$ gauge groups. Suppose that these two stacks of D6-branes intersect each other on $T_{i}^{2}$. Their intersecting number on $T_{i}^{2}$ is obtained by

$$
I_{a b}^{(i)}=\left(n_{a}^{i} m_{b}^{i}-m_{a}^{i} n_{b}^{i}\right),
$$

and their total intersecting number on $T^{6}$ is obtained by

$$
\left[\Pi_{a}\right] \cdot\left[\Pi_{b}\right]=I_{a b}=\prod_{i=1}^{3} I_{a b}^{(i)} .
$$

Then, chiral matter fields with bi-fundamental representations $\left(N_{a}, \bar{N}_{b}\right)_{(1,-1)}$ under $\mathrm{U}\left(N_{a}\right) \times \mathrm{U}\left(N_{b}\right)$ appear at intersecting points on $T_{i}^{2}$, where the index $(1,-1)$ denotes $\mathrm{U}(1)^{2}$ charges inside $\mathrm{U}\left(N_{a}\right)$ and $\mathrm{U}\left(N_{b}\right)$. There appear $I_{a b}$ families of bi-fundamental matter fields. When $I_{a b}$ is negative, there appear $\left|I_{a b}\right|$ families of matter fields with the conjugate representation $\left(\bar{N}_{a}, N_{b}\right)_{(-1,1)}$.

The total flavor symmetry is a direct product of flavor symmetries appearing on one of $T_{i}^{2}$. Thus, we concentrate on the flavor symmetry realized on one of $T_{i}^{2}$. Then, we denote $I_{a b}^{(i)}=g$. Theses modes on $T_{i}^{2}$ have definite $Z_{g}$ charges and $Z_{g}$ transformation is represented by

$$
Z=\left(\begin{array}{llll}
1 & & & \\
& \rho & & \\
& & \rho^{2} & \\
& & \ddots & \\
& & & \rho^{g-1}
\end{array}\right),
$$

where $\rho=e^{2 \pi i / g}$. In addition, there is a cyclic permutation symmetry $Z_{g}^{(C)}$ among these modes, i.e.

$$
C=\left(\begin{array}{cccccc}
0 & 1 & 0 & 0 & \cdots & 0 \\
0 & 0 & 1 & 0 & \cdots & 0 \\
& & & & \ddots & \\
1 & & & \cdots & 0
\end{array}\right) .
$$

Furthermore, these elements do not commute each other,

$$
C Z=\rho Z C .
$$

Thus, this flavor symmetry includes another $Z_{g}^{\prime}$ symmetry, which is represented by

$$
Z^{\prime}=\left(\begin{array}{lll}
\rho & & \\
& \ddots & \\
& & \rho
\end{array}\right) .
$$

Then, these would generate the non-Abelian flavor symmetry, $\left(Z_{g} \times Z_{g}^{\prime}\right) \rtimes Z_{g}^{(C)}$. 
For example, when $g=2$ and $g=3$, the symmetries correspond to $D_{4}$ and $\Delta(27)$. In addition, when the totally D-brane system has the $Z_{2}$ reflection symmetry $P$ between $i$-th mode and $(g-i)$-th mode the $\Delta(27)$ symmetry for $g=3$ is enhanced into $\Delta(54)$ [18].

Similarly, we can discuss models with more than two sets of D-branes. For example, suppose that we add $N_{c}$ stacks of D-branes to the above system, and that their intersecting numbers satisfy G.C.D. $\left(I_{a b}^{(i)}, I_{a c}^{(i)}, I_{b c}^{(i)}\right)=d$. Then, this model has the discrete flavor symmetry $\left(Z_{d} \times Z_{d}^{\prime}\right) \rtimes Z_{d}^{(C)}$.

The above result is applicable to intersecting D-brane models on orientifolds through simple extension. Also, we can extend our discussions to orbifold cases [18-22].

Since magnetized D-brane models are T-duals to intersecting D-brane models, the magnetized D-brane models also have the same discrete flavor symmetries. For example, we start with $\left(N_{a}+N_{b}\right)$ stacks of D9-branes on $T^{6}$. Then, we introduce the magnetic flux on $T_{i}^{2}$ along $\mathrm{U}(1)_{a}$ and $\mathrm{U}(1)_{b}$ directions in $\mathrm{U}\left(N_{a}+N_{b}\right)$ as

$$
F^{(i)}=2 \pi\left(\begin{array}{cccccc}
M_{a}^{(i)} & & & & & \\
& \ddots & & & & \\
& & M_{a}^{(i)} & & & \\
& & M_{b}^{(i)} & & \\
& & & \ddots & \\
& & & & M_{b}^{(i)}
\end{array}\right)
$$

where $M_{a}^{(i)}$ and $M_{b}^{(i)}$ are integers. This magnetic flux background breaks the gauge group $\mathrm{U}\left(N_{a}+N_{b}\right)$ into $\mathrm{U}\left(N_{a}\right) \times \mathrm{U}\left(N_{b}\right)$. The gaugino fields in the off-diagonal part correspond to the $\left(N_{a}, \bar{N}_{b}\right)$ bi-fundamental matter fields under the unbroken $\mathrm{U}\left(N_{a}\right) \times \mathrm{U}\left(N_{b}\right)$ gauge symmetry. Zero-modes with such representation appear in this model, and the number of zero-modes on $T_{i}^{2}$ is equal to $M_{a}^{(i)}-M_{b}^{(i)}$. When we denote $M_{a}^{(i)}-M_{b}^{(i)}=g$, this magnetized D-brane model leads to the same discrete flavor symmetry, $\left(Z_{g} \times Z_{g}^{\prime}\right) \rtimes Z_{g}^{(C)}$ as the above intersecting D-brane model.

\section{Discrete anomalies}

In this section, we study anomalies of discrete flavor symmetries.

\section{$3.1 \quad \mathrm{U}(1)$ anomalies}

Before studying anomalies of discrete flavor symmetries, it is useful to review anomalies of $\mathrm{U}(1)$ gauge symmetries. In this subsection, we give a brief review on $\mathrm{U}(1)$ anomalies $[13,14,56]$ (see also $[9,17]$ ).

First of all, we consider the torus compactification. A D6-brane has a charge of RR 7-form $C_{7}$. The total charge should vanish in a compact space. That leads to the following tadpole cancellation condition,

$$
\sum_{a} N_{a}\left[\Pi_{a}\right]=0
$$


The $\mathrm{SU}\left(N_{a}\right)^{3}$ anomaly coefficient is calculated in the intersecting D-brane models by

$$
A_{a}=\sum_{b} I_{a b} N_{b}
$$

because there are $I_{a b}$ matter fields with $\left(N_{a}, \bar{N}_{b}\right)_{(1,-1)}$ for $I_{a b}>0$ and $\left|I_{a b}\right|$ matter fields with $\left(\bar{N}_{a}, N_{b}\right)_{(-1,1)}$ for $I_{a b}<0$. However, the tadpole cancellation condition leads to

$$
\left[\Pi_{a}\right] \cdot \sum_{b} N_{b}\left[\Pi_{b}\right]=0
$$

That implies that $A_{a}=0$, that is, anomaly free.

The $\mathrm{U}(1)_{a} \times \mathrm{SU}\left(N_{b}\right)^{2}$ mixed anomaly coefficient is obtained by

$$
A_{a b}=N_{a} I_{a b}
$$

This anomaly is not always vanishing. However, this anomaly can always be canceled by the Green-Schwarz mechanism, where an axion shifts under the U(1) gauge transformation and the anomalous $\mathrm{U}(1)$ gauge boson becomes massive.

The U(1)-gravity ${ }^{2}$ anomaly coefficient is obtained by

$$
A_{a-\text { grav }}=N_{a} \sum_{b} I_{a b} N_{b} .
$$

This anomaly is always vanishing when the tadpole cancellation condition is satisfied.

Next, we review on anomalies for the orientifold compactification. That is, we introduce O6-branes along the direction $\prod_{i}\left[a_{i}\right]$. The system must be symmetric under the $Z_{2}$ reflection, $y_{i} \rightarrow-y_{i}$. In this case, we have to introduce a mirror $D 6_{a^{\prime}}$-branes with the winding number $\left(n_{a}^{i},-m_{a}^{i}\right)$ corresponding to $\left(n_{a}^{i}, m_{a}^{i}\right)$. The O6-brane has $(-4)$ times as RR charge as a D6-brane. Then, the RR-tadpole cancellation condition requires

$$
\begin{aligned}
\sum_{a} N_{a}\left(\left[\Pi_{a}\right]+\left[\Pi_{a^{\prime}}\right]\right)-4\left[\Pi_{O 6}\right] & =0 . \\
\sum_{a \neq b} N_{a}\left[\Pi_{b}\right] \cdot\left(\left[\Pi_{a}\right]+\left[\Pi_{a^{\prime}}\right]\right)+N_{b}\left[\Pi_{b}\right] \cdot\left[\Pi_{b^{\prime}}\right]-4\left[\Pi_{b}\right] \cdot\left[\Pi_{O 6}\right] & =0 .
\end{aligned}
$$

In addition to $I_{a b}$ families of $\left(N_{a}, \bar{N}_{b}\right)_{(1,-1)}$ matter fields, there appear $I_{a b^{\prime}}$ families of $\left(N_{a}, N_{b}\right)_{(1,1)}$ matter fields. Moreover, there appear matter fields with symmetric and asymmetric representations under $\mathrm{U}\left(N_{a}\right)$ with charge 2 . Their numbers are obtained by

$$
\begin{aligned}
\#_{a, \text { asymm }} & =\frac{1}{2}\left(\left[\Pi_{a}\right] \cdot\left[\Pi_{a^{\prime}}\right]-\left[\Pi_{a}\right] \cdot\left[\Pi_{O 6}\right]\right)+\left[\Pi_{a}\right] \cdot\left[\Pi_{O 6}\right], \\
\#_{a, \text { symm }} & =\frac{1}{2}\left(\left[\Pi_{a}\right] \cdot\left[\Pi_{a^{\prime}}\right]-\left[\Pi_{a}\right] \cdot\left[\Pi_{O 6}\right]\right) .
\end{aligned}
$$

In this case, we can show that the $\mathrm{SU}\left(N_{a}\right)^{3}$ anomaly coefficient always vanishes when the RR-tadpole cancellation condition is satisfied, similarly to in the torus compactification. Also, the $\mathrm{U}(1)_{a}-\mathrm{SU}\left(N_{b}\right)^{2}$ anomaly coefficient is not always vanishing, but such anomaly can be canceled by the Green-Schwarz mechanism. 
Finally, the $\mathrm{U}(1)_{a}$ - gravity ${ }^{2}$ anomaly coefficient is obtained by

$$
\begin{aligned}
A_{a-\text { grav }}= & \prod_{b \neq} N_{a} N_{b}\left(\left[\Pi_{a}\right] \cdot\left[\Pi_{b}\right]+\left[\Pi_{a}\right] \cdot\left[\Pi_{b^{\prime}}\right]\right)+2 \frac{N_{a}\left(N_{a}-1\right)}{2} \#_{a, \text { asymm }} \\
& +2 \frac{N_{a}\left(N_{a}+1\right)}{2} \#_{a, \text { symm }} \\
= & 3 N_{a}\left[\Pi_{a}\right] \cdot\left[\Pi_{O 6}\right] .
\end{aligned}
$$

This does not always vanish, but such anomaly can be canceled by the Green-Schwarz mechanism.

\subsection{Discrete anomalies}

In the gauge theory with the gauge group $G$ and the Abelian discrete symmetry $Z_{N}$, the $Z_{N}-G^{2}$ mixed anomaly coefficient is calculated by $[2-4,48,57,58]$,

$$
A_{Z_{N}-G^{2}}=\sum_{m} q^{(m)} T_{2}\left(\mathbf{R}^{(m)}\right)
$$

where the summation of $m$ is taken over fermions with $Z_{N}$ charges $q^{(m)}$ and the representation $\mathbf{R}^{(m)}$ under $G$. Here, $T_{2}\left(\mathbf{R}^{(m)}\right)$ denotes the Dynkin index and we use the normalization such that $T_{2}=1 / 2$ for the fundamental representation of $\mathrm{SU}(N)$. When the following condition is satisfied [2-4, 48, 57, 58],

$$
\sum_{m} q^{(m)} T_{2}\left(\mathbf{R}^{(m)}\right)=0(\bmod \quad N / 2)
$$

the $Z_{N}$ symmetry is anomaly-free. Similarly, we can calculate the $Z_{N}$-gravity ${ }^{2}$ anomaly coefficient by $\operatorname{Tr} q^{(m)}$. If $\operatorname{Tr} q^{(m)}=0(\bmod N / 2), Z_{N}$ is anomaly-free. For example, $Z_{2}$ symmetry is always anomaly-free.

Each generator of non-Abelian discrete symmetries corresponds to an Abelian symmetry. Thus, if each Abelian generator of non-Abelian discrete flavor symmetry satisfies the above anomaly-free condition, the total non-Abelian symmetry is anomaly-free. When some discrete Abelian symmetries are anomalous, the total non-Abelian discrete symmetry is broken, and the subgroup, which does not include anomalous generators, remains unbroken.

In the non-Abelian discrete symmetry, there appear multiplets and each generator is represented by a matrix, $M$. When $\operatorname{det} M=1$, the corresponding Abelian discrete symmetry is always anomaly-free. Only multiplets with $\operatorname{det} M \neq 1$ can contribute on anomalies. Since we have $\operatorname{det} Z^{\prime}=1$, the corresponding $Z_{g}^{\prime}$ symmetry is always anomalyfree. On the other hand, we find $\operatorname{det} Z=\operatorname{det} C=1$ for $g=\operatorname{odd}$ and $\operatorname{det} Z=\operatorname{det} C=-1$ for $g=$ even. That means that the discrete flavor symmetry $\left(Z_{g} \times Z_{g}^{\prime}\right) \rtimes Z_{g}^{(C)}$ is always anomaly-free for $g=$ odd, but $Z_{g}$ and $Z_{g}^{(C)}$ can be anomalous for $g=$ even. In particular, their $Z_{2}$ parts are anomalous. One has to check the anomaly-free condition for such $Z_{2}$ part for $Z_{g}$ and $Z_{g}^{(C)}$. For example, the $\Delta(27)$ flavor symmetry for $g=3$ is always anomaly-free. However, $Z_{2}$ subgroups of $D_{4}$ for $g=2$ corresponding to the following elements,

$$
\left(\begin{array}{cc}
1 & 0 \\
0 & -1
\end{array}\right), \quad\left(\begin{array}{ll}
0 & 1 \\
1 & 0
\end{array}\right),
$$


can be anomalous.

First, we discuss the torus compactification. For simplicity, we concentrate on the flavor symmetry appearing the first torus $T_{1}^{2}$ and we assume that all of intersecting numbers on $T_{1}^{2}, I_{a b}^{1}$, are even. Thus, the total flavor symmetry includes the $Z_{2}$ symmetry as well as $Z_{2}^{(C)}$, which can be anomalous. Also, we assume that there appears a trivial symmetry from the other $T_{2}^{2} \times T_{3}^{2}$. Now, let us examine the $Z_{2}-\mathrm{SU}\left(N_{a}\right)^{2}$ anomaly. There are $I_{a b}$ bi-fundamental matter fields with the representation $\left(N_{a}, \bar{N}_{b}\right)$. A half of $I_{a b}$ matter fields have even $Z_{2}$ charge and the others have odd $Z_{2}$ charge. The anomaly coefficient of $Z_{2}-\mathrm{SU}\left(N_{a}\right)^{2}$ anomaly can be written by

$$
\sum_{b} \frac{I_{a b}}{2} N_{b} \frac{1}{2}
$$

It vanishes because the tadpole cancellation condition, $\sum_{b} I_{a b} N_{b}=0$. Thus, this $Z_{2}$ symmetry is anomaly-free on the torus compactification. Since only this $Z_{2}$ symmetry can be anomalous and the others are always anomaly-free, the non-Abelian flavor symmetries $\left(Z_{g} \times Z_{g}^{\prime}\right) \rtimes Z_{g}^{(C)}$ are always anomaly-free in the torus compactification.

Next, we study the orientifold compactification. Similarly, we can calculate the $Z_{2}-\mathrm{SU}\left(N_{a}\right)^{2}$ anomaly coefficient,

$$
\sum_{b \neq a}\left(\frac{I_{a b}}{2} N_{b}+\frac{I_{a b^{\prime}}}{2} N_{b^{\prime}}\right) \frac{1}{2}+\frac{N_{a}-2}{4} \#_{a, \text { asymm }}+\frac{N_{a}+2}{4} \#_{a, \text { symm }}=\frac{\left[\Pi_{a}\right] \cdot\left[\Pi_{O 6}\right]}{2}
$$

That is not always vanishing, but it is proportional to the $\mathrm{U}(1)_{a}$-grav ${ }^{2}$ anomaly. Thus, this anomaly could be canceled when one requires the axion shift under the $Z_{2}$ transformation, which is related with the axion shift under $\mathrm{U}(1)_{a}$. In addition, when $\mathrm{D} 6_{a}$ branes are parallel to the O6-branes, $Z_{2}-\mathrm{SU}\left(N_{a}\right)^{2}$ anomaly coefficient is always vanishing.

\section{Majorana neutrino masses}

In the previous section, we have studied on anomalies of discrete flavor symmetries. Certain symmetries are anomaly-free. For example, the $\Delta(27)$ flavor symmetry is anomaly-free. Anomalous symmetries can be broken by non-perturbative effects. There is no guarantee that anomaly-free symmetries are not broken by stringy non-perturbative effects. In this section, we consider D-brane instanton effects as concrete non-perturbative effects. We study which form of right-handed Majorana neutrino mass matrix can be generated by D-brane instanton effects. Indeed, following [50-52], we study the sneutrino mass matrix assuming that the neutrino mass matrix has the same form and supersymmetry breaking effects are small.

\subsection{Neutrino mass matrix}

Here, we study right-handed Majorana neutrino masses, which can be generated by D-brane instanton effects. We assume that $g$ families of right-handed neutrinos $\nu_{R}^{a}$ appear by intersections between $\mathrm{D} 6_{c}$-brane and $\mathrm{D} 6_{d}$ branes, and that their intersecting numbers are equal 
to $I_{c d}^{(i)}=g$ for the i-th $T^{2}$ and $I_{c d}^{(j)}=1$ for the other tori. For the moment, let us concentrate on the three-generation model, $I_{c d}=3$, which can be obtained by $\left(I_{c d}^{(1)}, I_{c d}^{(2)}, I_{c d}^{(3)}\right)=(\underline{3,1,1})$, where the underline denotes all the possible permutations. We consider D2-brane instanton, which wraps one-cycle of each $T^{2}$ of $T^{6}=T^{2} \times T^{2} \times T^{2}$. We call it $D 2_{M}$-brane. It intersects with $\mathrm{D} 6_{c}$ brane and $\mathrm{D} 6_{d}$ brane. At these intersecting points, zero-modes $\alpha_{i}$ and $\gamma_{j}$ appear and their numbers are obtained by $I_{M c}$ and $I_{d M}$. Only if there are two zero-modes for both $\alpha_{i}$ and $\gamma_{j}$ the neutrino masses can be generated by D2-brane instanton effect [50-52],

$$
\begin{aligned}
M \int d^{2} \alpha d^{2} \gamma e^{-d_{a}^{i j} \alpha_{i} \nu_{R}^{a} \gamma_{j}} & =M c_{a b}, \\
c_{a b} & =\nu_{R}^{a} \nu_{R}^{b}\left(\varepsilon_{i j} \varepsilon_{k \ell} d_{a}^{i k} d_{b}^{j \ell}\right),
\end{aligned}
$$

where the mass scale $M$ would be determined by the string scale $M_{s t}$ and the instanton world volume $V$ as $M=M_{s t} e^{-V}$. Here, $d_{a}^{i j}$ is the 3-point coupling coefficient among $\alpha_{i}, \nu_{R}^{a}$ and $\gamma_{j}$ [33-36], which we show explicitly in the next subsection. The 3-point coupling coefficient $d_{a}^{i j}$ can be written by $d_{a}^{i j}=d_{a 1}^{i j} d_{a 2}^{i j} d_{a 3}^{i j}$, where $d_{a k}^{i j}$ for $k=1,2,3$ is the contribution from the $k$-th torus. In addition, when $\alpha_{i}, \gamma_{j}$, or $\nu^{a}$ are localized at a single intersecting point on the $k$-th torus, we omit the indexes such as $d_{a k}^{j}, d_{a k}^{i}$, or $d_{k}^{i j}$.

We have to take into account all of the possible $D 2_{M}$-brane configurations, which can generate the above neutrino mass terms. One can obtain two zero-modes of $\alpha_{i}$ and $\gamma_{j}$ for the $D 2_{M}$-brane set corresponding to $\mathrm{Sp}(2)$ or $\mathrm{U}(2)$ gauge group with the intersecting numbers $\left|I_{M c}\right|=\left|I_{M d}\right|=1[53,54]$ or a single $D 2_{M}$-brane with the intersecting numbers, $\left|I_{M c}\right|=\left|I_{M d}\right|=2$.

When the $D 2_{M}$-brane set corresponds to the $\mathrm{Sp}(2)$ or $\mathrm{U}(2)$ brane, the zero-modes, $\alpha_{i}$ and $\gamma_{j}$, are doublets and the gauge invariance allows the certain couplings, say $\alpha_{i}$ and $\gamma_{i}$, but not $\alpha_{i}$ and $\gamma_{j}$ for $i \neq j$. When $I_{M c}=I_{d M}=1$, the following form of the Majorana mass is generated,

$$
\int d^{2} \alpha d^{2} \gamma e^{-d_{a}^{11} \alpha_{1} \nu_{R}^{a} \gamma_{1}-d_{a}^{22} \alpha_{2} \nu_{R}^{a} \gamma_{2}}=\nu_{R}^{a} \nu_{R}^{b} d_{a}^{11} d_{b}^{22}
$$

More explicitly, the following form of mass matrix is obtained [53, 54],

$$
M c_{a b}=\left(\begin{array}{llll}
d_{1}^{11} d_{1}^{22} & d_{1}^{11} & d_{2}^{22} & d_{1}^{11} d_{3}^{22} \\
d_{2}^{11} d_{1}^{22} & d_{2}^{11} d_{2}^{22} & d_{2}^{11} d_{3}^{22} \\
d_{3}^{11} d_{1}^{22} & d_{3}^{11} d_{2}^{22} & d_{3}^{11} d_{3}^{22}
\end{array}\right)
$$

This Majorana mass matrix has the rank one. However, we have to take into account all of the $D 2_{M}$-brane configurations, that is, the position of $D 2_{M}$-brane sets. Thus, we integrate over the position of the $D 2_{M}$-brane sets. Such integration over the $D 2_{M}$-brane position would recover the cyclic permutation symmetry, $Z_{g=3}^{(C)}$. Then, we would obtain the following form of Majorana neutrino mass matrix,

$$
M=\left(\begin{array}{lll}
A & B & B \\
B & A & B \\
B & B & A
\end{array}\right)
$$


We will show this form by an explicit calculation in the next subsection. As a result, there remains the cyclic permutation symmetry, $Z_{g=3}^{(C)}$, unbroken, but $Z_{g=3}$ and $Z_{g=3}^{\prime}$ symmetries are broken by D-brane instanton effects, which generate the Majorana neutrino masses. This form also has the $Z_{2}$ reflection symmetry $P$. Thus, if the full D-brane system has the $Z_{2}$ reflection symmetry, the symmetry is enhanced into $S_{3}$.

Similarly, we can study a single $D 2_{M}$-brane with the intersecting numbers, $\left|I_{M c}\right|=$ $\left|I_{M d}\right|=2$. There are two types of $D 2_{M}$-brane instanton configurations leading to $\left|I_{M c}\right|=$ $\left|I_{M d}\right|=2$. In one type, we have the configuration with $\left|I_{M c}^{(j)}\right|=\left|I_{M d}^{(k)}\right|=2$ for $j \neq k$, and in the other type we have the configuration with $\left|I_{M c}^{(j)}\right|=\left|I_{M d}^{(j)}\right|=2$.

In the first case with $\left|I_{M c}^{(j)}\right|=\left|I_{M d}^{(k)}\right|=2$ for $j \neq k$, let us set e.g. $j=1$ and $k=2$. Then, the Yukawa coupling $d_{a}^{i j}$ can be written by $d_{a}^{i j}=d_{a 1}^{i} d_{a 2}^{j} d_{a 3}$. Also we assume that $I_{c d}^{(1)}=3$ and $I_{c d}^{(2)}=I_{c d}^{(3)}=1$. Then, the neutrino mass can be written by

$$
\varepsilon_{i j} \varepsilon_{k \ell} d_{a}^{i k} d_{b}^{j l}=\varepsilon_{i j} \varepsilon_{k l} d_{a 1}^{i} d_{2}^{k} d_{3} d_{b 1}^{j} d_{2}^{\ell} d_{3} .
$$

However, this vanishes identically [52]. We obtain the same result for $\left|I_{M c}^{(j)}\right|=\left|I_{M d}^{(k)}\right|=2$ with $j \neq k$, when $\left(I_{c d}^{(1)}, I_{c d}^{(2)}, I_{c d}^{(3)}\right)=(\underline{3,1,1})$.

On the other hand, if a single $\overline{D 2_{M}}$-brane configuration with $\left|I_{M c}^{(j)}\right|=\left|I_{M d}^{(j)}\right|=2$ is possible, we obtain the non-vanishing neutrino mass matrix $M c_{a b}$. Then, when we integrate over the position of the $D 2_{M}$-brane instanton, we would obtain the same results as eq. (4.4). Thus, the cyclic permutation symmetry $Z_{g=3}^{(C)}$ is recovered.

This result can be extended for models with $g$ flavors of neutrinos. When we take into account all of the possible D-brane instanton configurations, we would realize the neutrino mass matrix $M c_{a b}$ with the cyclic permutation symmetry $Z_{g}^{(C)}$, i.e.

$$
c_{a b}=c_{a^{\prime} b^{\prime}} \quad \text { for } \quad a^{\prime}=a+1, b^{\prime}=b+1 .
$$

Also the mass matrix is symmetric, i.e. $c_{a b}=c_{b a}$. For example, we obtain

$$
c_{a b}=\left(\begin{array}{cc}
A & B \\
B & A
\end{array}\right),
$$

for $g=2$ and

$$
c_{a b}=\left(\begin{array}{cccc}
A & B & B^{\prime} & B \\
B & A & B & B^{\prime} \\
B^{\prime} & B & A & B \\
B & B^{\prime} & B & A
\end{array}\right),
$$

for $g=4$. It is found that the D-brane instantons break $Z_{g}^{\prime}$ into $Z_{2}$ if $g$ is even. Otherwise, the $Z_{g}^{\prime}$ symmetry as well as the $Z_{g}$ symmetry is completely broken. However, the cyclic permutation symmetry remains. ${ }^{3}$

We have studied the neutrino mass matrix by assuming that the neutrino and sneutrino have the same mass matrix and supersymmetry breaking effect is small [50-52]. The important point to derive our result is the cyclic permutation symmetry. Thus, we would obtain the same result if the D-brane instatons do not break such a symmetry but supersymmetry is broken.

\footnotetext{
${ }^{3}$ These forms also have the $Z_{2}$ reflection symmetry.
} 


\subsection{Explicit computation}

Here, we discuss the Majorana neutrino mass matrix by computing explicitly the threegeneration models. We consider the D2-brane instanton corresponding to $\mathrm{Sp}(2)$ or $\mathrm{U}(2)$ gauge symmetry. Suppose that $\mathrm{D} 6_{c}$ and $\mathrm{D} 6_{d}$ branes have the intersecting number, $I_{c d}=3$, and at three intersecting points there appear three generations of right-handed neutrinos. We set $\left(I_{c d}^{(1)}, I_{c d}^{(2)}, I_{c d}^{3)}\right)=(3,1,1)$, and $I_{M c}=I_{d M}=1$. Because the right-handed neutrinos are localized at different points from each other on the first torus, only the first torus is important for the flavor symmetry. Thus, we concentrate on the first torus for computations on Yukawa couplings and Majorana masses. We also omit the index corresponding to the $k$-torus. In the following computations, we set Wilson line moduli zero because it dose not affect the flavor structure (see appendix A for more detail).

There are three generations of $\nu_{a}$ and we here label their flavor index as $a=0,1,2$. Also there are two-zero modes, $\alpha_{i}$ and $\gamma_{j}(i, j=1,2)$, but note that these indexes $i, j$ correspond to the doublets under $\mathrm{Sp}(2)$ or $\mathrm{U}(2)$ and the intersecting numbers, $I_{c M}$, and $I_{M d}$, are equal to one, $I_{c M}=I_{M d}=1$.

Suppose that there are three fields $\phi_{a}, \chi_{i^{\prime}}$ and $\chi_{j^{\prime}}$ with the "flavor numbers", $a=$ $0, \cdots, I_{c d}-1, i^{\prime}=0, \cdots, I_{d M}-1$, and $j^{\prime}=0, \cdots, I_{M c}-1$, where $I_{c d}, I_{d M}$, and $I_{M c}$ are the corresponding intersecting numbers on the torus. In this case, the 3 -point couplings $d_{a}^{i^{\prime} j^{\prime}}$ among three fields can be calculated by [33-36]

$$
d_{a}^{i^{\prime} j^{\prime}}=C \sum_{\ell \in Z} \exp \left(\frac{-A_{a i^{\prime} j^{\prime}}(\ell)}{2 \pi \alpha^{\prime}}\right)
$$

where $C$ is a flavor-independent constant due to quantum contributions and

$$
A_{a i^{\prime} j^{\prime}}(\ell)=\frac{1}{2} A\left|I_{c d} I_{d M} I_{M c}\right|\left(\frac{a}{I_{c d}}+\frac{i^{\prime}}{I_{d M}}+\frac{j^{\prime}}{I_{M c}}+\frac{\varepsilon}{I_{d M} I_{M c}}+\ell\right)^{2},
$$

and $A$ denotes the area of the first torus. Here, $\varepsilon$ denotes the position of $D 2_{M}$-brane on the first torus and we normalize $\varepsilon$ such that $\varepsilon$ varies $[0,1]$ on the torus. Note that this coupling corresponds to the contribution on the first torus, which determines the flavor structure, but we have omitted the index corresponding to the first torus.

By using the $\vartheta$-function,

$$
\vartheta\left[\begin{array}{l}
a \\
b
\end{array}\right](\nu, \tau)=\sum_{\ell \in Z} \exp \left[\pi i(a+\ell)^{2} \tau+2 \pi i(a+\ell)(\nu+b)\right],
$$

we can write

$$
d_{a}^{i^{\prime} j^{\prime}}=C \vartheta\left[\begin{array}{c}
\frac{a}{I_{c d}}+\frac{i^{\prime}}{I_{d M}}+\frac{j^{\prime}}{I_{M c}}+\frac{\varepsilon}{I_{d M} I_{M c}} \\
0
\end{array}\right]\left(0, \frac{i A\left|I_{c d} I_{d M} I_{M c}\right|}{4 \pi^{2} \alpha^{\prime}}\right) .
$$

Our model corresponds to $a=0,1,2, I_{c d}=3, i^{\prime}=j^{\prime}=0, I_{d M}=I_{M c}=1$. In the above model, the 3 -point couplings among $\nu_{a}, \alpha_{i}$, and $\gamma_{j}$ are written by

$$
d_{a}^{i j}=\delta_{i j} \vartheta\left[\begin{array}{c}
-\frac{a}{3}+\varepsilon \\
0
\end{array}\right]\left(0, \frac{3 i A}{4 \pi^{2} \alpha^{\prime}}\right) \text {. }
$$


Recall again that the indexes $i$ and $j$ of $\alpha_{i}$ and $\gamma_{j}$ are doublet indexes under $\operatorname{Sp}(2)$ or $\mathrm{U}(2)$.

Using this, the matrix $c_{a b}$ is written by the integration of the position $\varepsilon$ over $[0,1]$,

$$
\begin{aligned}
c_{a b}= & \int_{0}^{1} d \varepsilon \vartheta\left[\begin{array}{c}
-\frac{a}{3}+\varepsilon \\
0
\end{array}\right]\left(0, \frac{3 i A}{4 \pi^{2} \alpha^{\prime}}\right) \vartheta\left[\begin{array}{c}
-\frac{b}{3}+\varepsilon \\
0
\end{array}\right]\left(0, \frac{3 i A}{4 \pi^{2} \alpha^{\prime}}\right) \\
= & \int_{0}^{1} d \varepsilon \sum_{m=1}^{2} \vartheta\left[\begin{array}{c}
-\frac{a}{6}-\frac{b}{6}+\varepsilon+\frac{m}{2} \\
0
\end{array}\right]\left(0, \frac{3 i A}{2 \pi^{2} \alpha^{\prime}}\right) \\
& \times \vartheta\left[\begin{array}{c}
-\frac{a}{6}+\frac{b}{6}+\frac{m}{2} \\
0
\end{array}\right]\left(0, \frac{3 i A}{2 \pi^{2} \alpha^{\prime}}\right) .
\end{aligned}
$$

We obtain

$$
\begin{aligned}
& \int_{0}^{1} d \varepsilon \vartheta\left[\begin{array}{c}
-\frac{a}{3}+\varepsilon+\frac{m}{2} \\
0
\end{array}\right]\left(0, \frac{3 i A}{2 \pi^{2} \alpha^{\prime}}\right) \\
& \quad=\int_{0}^{1} d \varepsilon \sum_{l \in Z} \exp \left[\pi i(-a / 3+\varepsilon+m / 2+\ell)^{2}\left(\frac{3 i A}{2 \pi^{2} \alpha^{\prime}}\right)\right] \\
& \quad=\int_{-\infty}^{\infty} d x \exp \left[-\frac{3 A}{2 \pi \alpha^{\prime}}(x-a / 3+m / 2)^{2}\right] \\
& \quad=\sqrt{\frac{2 \pi^{2} \alpha^{\prime}}{3 A}}
\end{aligned}
$$

Using it, the matrix elements $c_{a b}$ can be computed as follows. It is found that the diagonal elements $c_{a a}$ do not depend on $a$ and they are written by

$$
c_{a a}=\sqrt{\frac{2 \pi^{2} \alpha^{\prime}}{3 A}}\left(\vartheta\left[\begin{array}{l}
0 \\
0
\end{array}\right]\left(0, \frac{3 i A}{2 \pi^{2} \alpha^{\prime}}\right)+\vartheta\left[\begin{array}{c}
\frac{1}{2} \\
0
\end{array}\right]\left(0, \frac{3 i A}{2 \pi^{2} \alpha^{\prime}}\right)\right) .
$$

Similarly, the off-diagonal elements are written by

$$
\begin{aligned}
& c_{01}=\sqrt{\frac{2 \pi^{2} \alpha^{\prime}}{3 A}}\left(\vartheta\left[\begin{array}{c}
\frac{1}{6} \\
0
\end{array}\right]\left(0, \frac{3 i A}{2 \pi^{2} \alpha^{\prime}}\right)+\vartheta\left[\begin{array}{c}
\frac{2}{3} \\
0
\end{array}\right]\left(0, \frac{3 i A}{2 \pi^{2} \alpha^{\prime}}\right)\right), \\
& c_{02}=\sqrt{\frac{2 \pi^{2} \alpha^{\prime}}{3 A}}\left(\vartheta\left[\begin{array}{c}
\frac{1}{3} \\
0
\end{array}\right]\left(0, \frac{3 i A}{2 \pi^{2} \alpha^{\prime}}\right)+\vartheta\left[\begin{array}{c}
\frac{5}{6} \\
0
\end{array}\right]\left(0, \frac{3 i A}{2 \pi^{2} \alpha^{\prime}}\right)\right), \\
& c_{12}=\sqrt{\frac{2 \pi^{2} \alpha^{\prime}}{3 A}}\left(\vartheta\left[\begin{array}{c}
\frac{1}{6} \\
0
\end{array}\right]\left(0, \frac{3 i A}{2 \pi^{2} \alpha^{\prime}}\right)+\vartheta\left[\begin{array}{c}
\frac{2}{3} \\
0
\end{array}\right]\left(0, \frac{3 i A}{2 \pi^{2} \alpha^{\prime}}\right)\right) .
\end{aligned}
$$

However, we have the following formula of the $\vartheta$-function

$$
\begin{gathered}
\vartheta\left[\begin{array}{l}
a \\
b
\end{array}\right](\nu, \tau)=\vartheta\left[\begin{array}{c}
a+1 \\
b
\end{array}\right](\nu, \tau), \\
\vartheta\left[\begin{array}{c}
-a \\
0
\end{array}\right](0, \tau)=\vartheta\left[\begin{array}{l}
a \\
0
\end{array}\right](0, \tau) .
\end{gathered}
$$




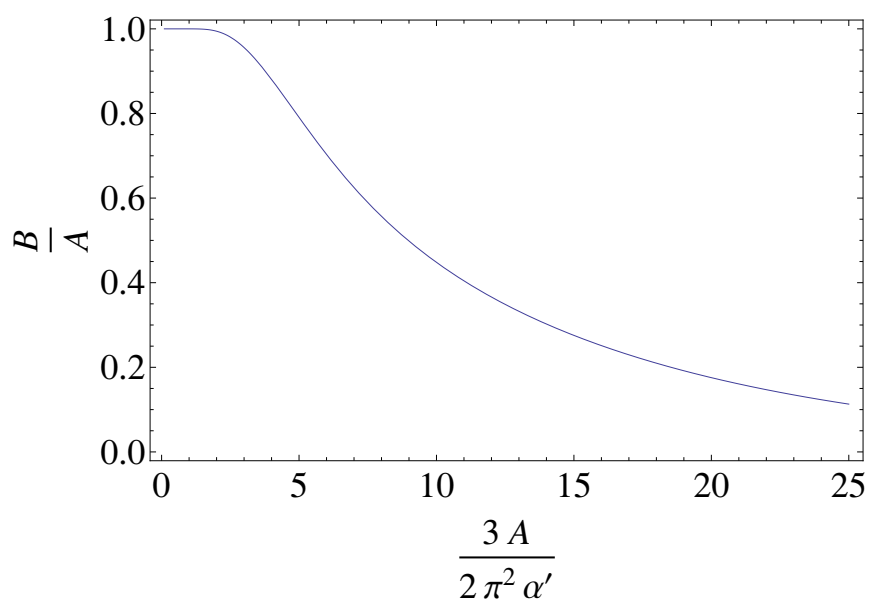

Figure 1. $B / A$ vs. $3 A / 2 \pi^{2} \alpha^{\prime}$.

Then, we see that all of the off-diagonal elements are the same,

$$
c_{01}=c_{12}=c_{20}
$$

That is, we can realize the form (4.4) by explicit calculations. Figure 1 shows the ratio $B / A=c_{12} / c_{a a}$ in (4.4) by varying the area $3 A / 2 \pi^{2} \alpha^{\prime}$.

\subsection{Phenomenological implication}

Here we discuss phenomenological implication of our result. The Majorana mass matrix with the form (4.4) can be diagonalized by the following matrix,

$$
\left(\begin{array}{ccc}
\sqrt{2 / 3} c & 1 / \sqrt{3} & -\sqrt{2 / 3} s \\
-1 / \sqrt{6} c-1 / \sqrt{2} s & 1 / \sqrt{3} & 1 / \sqrt{6} s-1 / \sqrt{2} c \\
-1 / \sqrt{6} c+1 / \sqrt{2} s & 1 / \sqrt{3} & 1 / \sqrt{6} s+1 / \sqrt{2} c
\end{array}\right),
$$

where $c=\cos \theta$ and $s=\sin \theta$, and the eigenvalues are $A-B, A+2 B$ and $A-B$. That is, two eigenvalues are degenerate. This is because the mass matrix (4.4) has the additional $Z_{2}$ reflection symmetry $P$ and the symmetry is enhanced into $S_{3}$. At any rate, this form of the mixing matrix is interesting, although the mass eigenvalues may be not completely realistic.

Suppose that the Dirac neutrino Yukawa couplings and charged lepton mass matrix are almost diagonal. ${ }^{4}$ Then, the lepton mixing matrix is obtained as the above matrix (4.23). That is the trimaximal matrix.

When $s=0$, the above matrix becomes the tri-bimaximal mixing matrix. In fieldtheoretical model building, the tri-bimaximal mixing matrix can be obtained as follows [15]. We start with a larger flavor symmetry and break by vacuum expectation values of scalar fields. However, one assumes that $Z_{3}$ and $Z_{2}$ subsymmetries remain in the charged lepton or neutrino mass terms. Then, the tri-bimaximal mixing matrix can be realized. In our string theory, such a $Z_{3}$ symmetry is realized by geometrical symmetry of the cyclic

\footnotetext{
${ }^{4}$ The $\Delta(27)$ flavor symmetry as well as $\Delta(54)$ flavor symmetry may be useful to realize such a form.
} 
permutation $Z_{3}^{C}$, which can not be broken by the D-brane instanton effects, although other symmetries are broken.

We may need some corrections to realize the experimental values of neutrino masses. ${ }^{5}$ At least, the above results show that we can realize non-trivial mixing in the lepton sector even though our assumption above the Dirac masses can not be realized.

\section{Conclusion and discussion}

We have studied the flavor structure in intersecting D-brane models. We have discussed the anomalies of flavor symmetries. Certain symmetries are anomaly-free, and anomaly coefficients of discrete symmetries have the specific feature. We have studied the Majorana neutrino masses, which can be generated by D-brane instanton effects. It is found that the mass matrix form with the cyclic permutation symmetry can be realized by integrating over the position of D-brane instanton. That would lead to the interesting form of mixing angles. It is interesting to apply our results for more concrete models. We would study numerical analyses elsewhere.

In some models, there appear more than one pair of Higgs fields. Their masses would be generated by D-brane instanton effects. It would be important to study the form of such Higgs mass matrix. Also, some of Yukawa couplings may be generated by D-brane instanton effects. Thus, it would be important to extend our analysis to Higgs mass matrix and Yukawa matrices.

\section{Acknowledgments}

The work of Y. H. is supported in part by the Grant-in-Aid for Japan Society for the Promotion of Science (JSPS) Fellows No.25-1107. The work of T.K. is supported in part by the Grants-in-Aid for Scientific No. 25400252 from the Ministry of Education, Culture,Sports, Science and Technology of Japan.

\section{A Integration of Wilson line moduli}

Here we integrate Wilson line moduli of the $D 2_{M}$-brane. Non-zero Wilson line varies the 3 point coupling (4.12) to the following form.

$$
d_{a}^{i^{\prime} j^{\prime}}=C \vartheta\left[\begin{array}{c}
\frac{a}{I_{c d}}+\frac{i^{\prime}}{I_{d M}}+\frac{j^{\prime}}{I_{M c}}+\frac{\varepsilon}{I_{d M} I_{M c}} \\
0
\end{array}\right]\left(\phi, \frac{i A\left|I_{c d} I_{d M} I_{M c}\right|}{4 \pi^{2} \alpha^{\prime}}\right) .
$$

Here, $\phi$ is Wilson line phase. The matrix $c_{a b}$ is written by the integration of the position $\varepsilon$ and Wilson line moduli $\phi$.

$$
c_{a b}=\int d \phi \int_{0}^{1} d \varepsilon \vartheta\left[\begin{array}{c}
-\frac{a}{3}+\varepsilon \\
0
\end{array}\right]\left(\phi, \frac{3 i A}{4 \pi^{2} \alpha^{\prime}}\right) \vartheta\left[\begin{array}{c}
-\frac{b}{3}+\varepsilon \\
0
\end{array}\right]\left(\phi, \frac{3 i A}{4 \pi^{2} \alpha^{\prime}}\right)
$$

\footnotetext{
${ }^{5}$ To resolve the degeneracy between two mass eigenvalues, it may be important to break the $Z_{2}$ reflection symmetry $P$. The full D-brane system, i.e. the full Lagrangian of the low-energy effective field theory, may not have such $Z_{2}$ symmetry and the above degeneracy may be resolved by radiative corrections.
} 


$$
\begin{aligned}
= & \int d \phi \int_{0}^{1} d \varepsilon \sum_{m=1}^{2} \vartheta\left[\begin{array}{c}
-\frac{a}{6}-\frac{b}{6}+\varepsilon+\frac{m}{2} \\
0
\end{array}\right]\left(2 \phi, \frac{3 i A}{2 \pi^{2} \alpha^{\prime}}\right) \\
& \times \vartheta\left[\begin{array}{c}
-\frac{a}{6}+\frac{b}{6}+\frac{m}{2} \\
0
\end{array}\right]\left(0, \frac{3 i A}{2 \pi^{2} \alpha^{\prime}}\right) .
\end{aligned}
$$

We get

$$
\begin{aligned}
& \int d \phi \int_{0}^{1} d \epsilon \vartheta\left[\begin{array}{c}
-\frac{a}{6}-\frac{b}{6}+\epsilon+\frac{m}{2} \\
0
\end{array}\right]\left(2 \phi, 2 i \frac{3 A}{2 \pi^{2} \alpha^{\prime}}\right) \\
& =\int d \phi \int_{0}^{1} d \epsilon \sum_{l \in \mathbf{Z}} e^{-\frac{3 A}{\pi \alpha^{\prime}}\left(-\frac{a}{6}-\frac{b}{6}+\epsilon+\frac{m}{2}+l\right)^{2}+4 \pi i\left(-\frac{a}{6}-\frac{b}{6}+\epsilon+\frac{m}{2}+l\right) \phi} \\
& =\int d \phi \int_{0}^{1} d \epsilon \sum_{l \in \mathbf{Z}} e^{-\frac{3 A}{\pi \alpha^{\prime}}\left(-\frac{a}{6}-\frac{b}{6}+\epsilon+\frac{m}{2}+l+i \frac{2 \pi^{2} \alpha^{\prime}}{3 A} \phi\right)^{2}-\frac{4 \pi^{3} \alpha^{\prime} \phi^{2}}{3 A}} \\
& =\sqrt{\frac{\pi^{2} \alpha^{\prime}}{3 A}} \int d \phi e^{-\frac{4 \pi^{3} \alpha^{\prime} \phi^{2}}{3 A}} .
\end{aligned}
$$

This factor is independent of flavor index, but universal. Thus, the integration of Wilson line moduli does not affect flavor structure.

Open Access. This article is distributed under the terms of the Creative Commons Attribution License (CC-BY 4.0), which permits any use, distribution and reproduction in any medium, provided the original author(s) and source are credited.

\section{References}

[1] G. Altarelli and F. Feruglio, Discrete flavor symmetries and models of neutrino mixing, Rev. Mod. Phys. 82 (2010) 2701 [arXiv: 1002.0211] [INSPIRE].

[2] H. Ishimori et al., Non-Abelian discrete symmetries in particle physics, Prog. Theor. Phys. Suppl. 183 (2010) 1 [arXiv:1003.3552] [INSPIRE].

[3] H. Ishimori, T. Kobayashi, H. Ohki, H. Okada, Y. Shimizu and M. Tanimoto, An introduction to non-Abelian discrete symmetries for particle physicists, Lect. Notes Phys. 858 (2012) 1 [INSPIRE].

[4] H. Ishimori et al., Non-Abelian discrete symmetry for flavors, Fortsch. Phys. 61 (2013) 441 [INSPIRE].

[5] S.F. King and C. Luhn, Neutrino mass and mixing with discrete symmetry, Rept. Prog. Phys. 76 (2013) 056201 [arXiv:1301.1340] [InSPIRE].

[6] P.F. Harrison, D.H. Perkins and W.G. Scott, Tri-bimaximal mixing and the neutrino oscillation data, Phys. Lett. B 530 (2002) 167 [hep-ph/0202074] [INSPIRE].

[7] P.F. Harrison and W.G. Scott, Symmetries and generalizations of tri-bimaximal neutrino mixing, Phys. Lett. B 535 (2002) 163 [hep-ph/0203209] [INSPIRE].

[8] P.F. Harrison and W.G. Scott, Permutation symmetry, tri-bimaximal neutrino mixing and the S3 group characters, Phys. Lett. B 557 (2003) 76 [hep-ph/0302025] [INSPIRE]. 
[9] L.E. Ibanez and A.M. Uranga, String theory and particle physics: an introduction to string phenomenology, Cambridge University Press, Cambridge U.K. (2012).

[10] C. Bachas, A way to break supersymmetry, hep-th/9503030 [INSPIRE].

[11] M. Berkooz, M.R. Douglas and R.G. Leigh, Branes intersecting at angles, Nucl. Phys. B 480 (1996) 265 [hep-th/9606139] [INSPIRE].

[12] R. Blumenhagen, L. Görlich, B. Körs and D. Lüst, Noncommutative compactifications of type-I strings on tori with magnetic background flux, JHEP 10 (2000) 006 [hep-th/0007024] [INSPIRE].

[13] G. Aldazabal, S. Franco, L.E. Ibáñez, R. Rabadán and A.M. Uranga, $D=4$ chiral string compactifications from intersecting branes, J. Math. Phys. 42 (2001) 3103 [hep-th/0011073] [INSPIRE].

[14] G. Aldazabal, S. Franco, L.E. Ibáñez, R. Rabadán and A.M. Uranga, Intersecting brane worlds, JHEP 02 (2001) 047 [hep-ph/0011132] [INSPIRE].

[15] C. Angelantonj, I. Antoniadis, E. Dudas and A. Sagnotti, Type I strings on magnetized orbifolds and brane transmutation, Phys. Lett. B 489 (2000) 223 [hep-th/0007090] [INSPIRE].

[16] L.E. Ibáñez, F. Marchesano and R. Rabadán, Getting just the Standard Model at intersecting branes, JHEP 11 (2001) 002 [hep-th/0105155] [INSPIRE].

[17] R. Blumenhagen, B. Körs, D. Lüst and S. Stieberger, Four-dimensional string compactifications with D-branes, orientifolds and fluxes, Phys. Rept. 445 (2007) 1 [hep-th/0610327] [INSPIRE].

[18] H. Abe, K.-S. Choi, T. Kobayashi and H. Ohki, Non-Abelian discrete flavor symmetries from magnetized/intersecting brane models, Nucl. Phys. B 820 (2009) 317 [arXiv:0904.2631] [INSPIRE].

[19] H. Abe, K.-S. Choi, T. Kobayashi and H. Ohki, Magnetic flux, Wilson line and orbifold, Phys. Rev. D 80 (2009) 126006 [arXiv:0907.5274] [inSPIRE].

[20] H. Abe, K.-S. Choi, T. Kobayashi and H. Ohki, Flavor structure from magnetic fluxes and non-Abelian Wilson lines, Phys. Rev. D 81 (2010) 126003 [arXiv:1001.1788] [INSPIRE].

[21] M. Berasaluce-Gonzalez, P.G. Camara, F. Marchesano, D. Regalado and A.M. Uranga, Non-Abelian discrete gauge symmetries in 4d string models, JHEP 09 (2012) 059 [arXiv:1206.2383] [INSPIRE].

[22] F. Marchesano, D. Regalado and L. Vazquez-Mercado, Discrete flavor symmetries in D-brane models, JHEP 09 (2013) 028 [arXiv: 1306.1284] [INSPIRE].

[23] T. Kobayashi, S. Raby and R.-J. Zhang, Searching for realistic 4d string models with a Pati-Salam symmetry: orbifold grand unified theories from heterotic string compactification on a $Z_{6}$ orbifold, Nucl. Phys. B 704 (2005) 3 [hep-ph/0409098] [INSPIRE].

[24] T. Kobayashi, H.P. Nilles, F. Ploger, S. Raby and M. Ratz, Stringy origin of non-Abelian discrete flavor symmetries, Nucl. Phys. B 768 (2007) 135 [hep-ph/0611020] [InSPIRE].

[25] P. Ko, T. Kobayashi, J.-H. Park and S. Raby, String-derived $D_{4}$ flavor symmetry and phenomenological implications, Phys. Rev. D 76 (2007) 035005 [Erratum ibid. D 76 (2007) 059901] [arXiv: 0704.2807] [INSPIRE]. 
[26] T. Higaki, N. Kitazawa, T. Kobayashi and K.-J. Takahashi, Flavor structure and coupling selection rule from intersecting D-branes, Phys. Rev. D 72 (2005) 086003 [hep-th/0504019] [INSPIRE].

[27] S. Hamidi and C. Vafa, Interactions on orbifolds, Nucl. Phys. B 279 (1987) 465 [INSPIRE].

[28] L.J. Dixon, D. Friedan, E.J. Martinec and S.H. Shenker, The conformal field theory of orbifolds, Nucl. Phys. B 282 (1987) 13 [INSPIRE].

[29] T.T. Burwick, R.K. Kaiser and H.F. Muller, General Yukawa couplings of strings on $Z_{N}$ orbifolds, Nucl. Phys. B 355 (1991) 689 [INSPIRE].

[30] J. Erler, D. Jungnickel, M. Spalinski and S. Stieberger, Higher twisted sector couplings of $Z_{N}$ orbifolds, Nucl. Phys. B 397 (1993) 379 [hep-th/9207049] [INSPIRE].

[31] K.-S. Choi and T. Kobayashi, Higher order couplings from heterotic orbifold theory, Nucl. Phys. B 797 (2008) 295 [arXiv:0711.4894] [INSPIRE].

[32] T. Kobayashi, S.L. Parameswaran, S. Ramos-Sanchez and I. Zavala, Revisiting coupling selection rules in heterotic orbifold models, JHEP 05 (2012) 008 [Erratum ibid. 12 (2012) 049] [arXiv: 1107.2137] [INSPIRE].

[33] M. Cvetič and I. Papadimitriou, Conformal field theory couplings for intersecting D-branes on orientifolds, Phys. Rev. D 68 (2003) 046001 [Erratum ibid. D 70 (2004) 029903] [hep-th/0303083] [INSPIRE].

[34] S.A. Abel and A.W. Owen, Interactions in intersecting brane models, Nucl. Phys. B 663 (2003) 197 [hep-th/0303124] [INSPIRE].

[35] D. Cremades, L.E. Ibáñez and F. Marchesano, Yukawa couplings in intersecting D-brane models, JHEP 07 (2003) 038 [hep-th/0302105] [INSPIRE].

[36] S.A. Abel and A.W. Owen, $N$ point amplitudes in intersecting brane models, Nucl. Phys. B 682 (2004) 183 [hep-th/0310257] [INSPIRE].

[37] D. Cremades, L.E. Ibáñez and F. Marchesano, Computing Yukawa couplings from magnetized extra dimensions, JHEP 05 (2004) 079 [hep-th/0404229] [INSPIRE].

[38] H. Abe, K.-S. Choi, T. Kobayashi and H. Ohki, Higher order couplings in magnetized brane models, JHEP 06 (2009) 080 [arXiv:0903.3800] [INSPIRE].

[39] Y. Hamada and T. Kobayashi, Massive modes in magnetized brane models, Prog. Theor. Phys. 128 (2012) 903 [arXiv:1207.6867] [INSPIRE].

[40] M. Berasaluce-Gonzalez, L.E. Ibáñez, P. Soler and A.M. Uranga, Discrete gauge symmetries in D-brane models, JHEP 12 (2011) 113 [arXiv:1106.4169] [INSPIRE].

[41] L.E. Ibáñez, A.N. Schellekens and A.M. Uranga, Discrete gauge symmetries in discrete MSSM-like orientifolds, Nucl. Phys. B 865 (2012) 509 [arXiv:1205.5364] [InSPIRE].

[42] M. Berasaluce-Gonzalez, P.G. Camara, F. Marchesano and A.M. Uranga, $Z_{p}$ charged branes in flux compactifications, JHEP 04 (2013) 138 [arXiv:1211.5317] [INSPIRE].

[43] P. Anastasopoulos, M. Cvetič, R. Richter and P.K.S. Vaudrevange, String constraints on discrete symmetries in MSSM type II quivers, JHEP 03 (2013) 011 [arXiv:1211.1017] [INSPIRE].

[44] G. Honecker and W. Staessens, To tilt or not to tilt: discrete gauge symmetries in global intersecting D-brane models, JHEP 10 (2013) 146 [arXiv:1303.4415] [INSPIRE]. 
[45] N.G. Cabo Bizet et al., $R$-charge conservation and more in factorizable and non-factorizable orbifolds, JHEP 05 (2013) 076 [arXiv:1301.2322] [INSPIRE].

[46] H.P. Nilles, S. Ramos-Sánchez, M. Ratz and P.K.S. Vaudrevange, A note on discrete $R$ symmetries in $Z_{6}$-II orbifolds with Wilson lines, Phys. Lett. B 726 (2013) 876 [arXiv: 1308.3435] [INSPIRE].

[47] N.G. Cabo Bizet et al., Discrete R-symmetries and anomaly universality in heterotic orbifolds, JHEP 02 (2014) 098 [arXiv: 1308.5669] [INSPIRE].

[48] T. Araki et al., (Non-)Abelian discrete anomalies, Nucl. Phys. B 805 (2008) 124 [arXiv: 0805.0207] [INSPIRE].

[49] T. Araki, K.-S. Choi, T. Kobayashi, J. Kubo and H. Ohki, Discrete R-symmetry anomalies in heterotic orbifold models, Phys. Rev. D 76 (2007) 066006 [arXiv:0705.3075] [InSPIRE].

[50] R. Blumenhagen, M. Cvetič and T. Weigand, Spacetime instanton corrections in $4 D$ string vacua: the seesaw mechanism for D-brane models, Nucl. Phys. B 771 (2007) 113 [hep-th/0609191] [INSPIRE].

[51] R. Blumenhagen, M. Cvetič, S. Kachru and T. Weigand, D-brane instantons in type II orientifolds, Ann. Rev. Nucl. Part. Sci. 59 (2009) 269 [arXiv:0902.3251] [InSPIRE].

[52] L.E. Ibáñez and A.M. Uranga, Neutrino Majorana masses from string theory instanton effects, JHEP 03 (2007) 052 [hep-th/0609213] [INSPIRE].

[53] L.E. Ibáñez, A.N. Schellekens and A.M. Uranga, Instanton induced neutrino Majorana masses in CFT orientifolds with MSSM-like spectra, JHEP 06 (2007) 011 [arXiv: 0704.1079] [INSPIRE].

[54] S. Antusch, L.E. Ibáñez and T. Macri, Neutrino masses and mixings from string theory instantons, JHEP 09 (2007) 087 [arXiv:0706.2132] [INSPIRE].

[55] M. Cvetič, R. Richter and T. Weigand, Computation of D-brane instanton induced superpotential couplings: Majorana masses from string theory, Phys. Rev. D 76 (2007) 086002 [hep-th/0703028] [InSPIRE].

[56] M. Cvetič, G. Shiu and A.M. Uranga, Chiral four-dimensional $N=1$ supersymmetric type $2 A$ orientifolds from intersecting D6 branes, Nucl. Phys. B 615 (2001) 3 [hep-th/0107166] [INSPIRE].

[57] L.E. Ibáñez and G.G. Ross, Discrete gauge symmetry anomalies, Phys. Lett. B 260 (1991) 291 [INSPIRE].

[58] T. Banks and M. Dine, Note on discrete gauge anomalies, Phys. Rev. D 45 (1992) 1424 [hep-th/9109045] [INSPIRE]. 